\title{
GROUP-BAY STOWAGE PLANNING PROBLEM FOR CONTAINER SHIP
}

\author{
Shen Yifan ${ }^{a}$ \\ Zhao Ning ${ }^{b}$ \\ Mi Weijian ${ }^{b}$ \\ a Scientific Research Academy, Shanghai Maritime University, 201306, Shanghai, CHINA \\ ${ }^{\mathrm{b}}$ Logistics Engineering College, Shanghai Maritime University, 201306, Shanghai, CHINA
}

\begin{abstract}
Stowage planning is the core of ship planning. It directly influences the seaworthiness of container ship and the handling efficiency of container terminal. As the latter step of container ship stowage plan, terminal stowage planning optimizes terminal cost according to pre-plan. Group-Bay stowage planning is the smallest sub problem of terminal stowage planning problem. A group-bay stowage planning model is formulated to minimize relocation, crane movement and target weight gap satisfying both ship owner and container terminal. A GA-A* hybrid algorithm is designed to solve this problem. Numerical experiment shown the validity and the efficiency.
\end{abstract}

Keywords: Container Terminal, Group-Bay Stowage Plan, Terminal Stowage Plan, Hybrid GA

\section{INTRODUCTION}

Despite the economic contraction of recent years, the container trade volume has been sustaining expanding. To meet the expanding demand of maritime transportation, container terminals has been focusing on management and decision evolution to enlarge their operation capability. Now, the research on the container terminal mainly focuses on two aspects, equipment automation and intelligent decisionmaking. There are quite a few scholars who have carried out some research work on these two points[3-4, 9-11]. In intelligent decision, container stowage plan has always been a major problem in terminal decision problems. As a core phase of ship planning, stowage planning affects equipment efficiency and energy consumption to a large extent. Since 1970s, stowage planning has been modeled and analyzed by experts and scholars from many scopes, such as decision making systems, mathematical modeling, simulation, intelligent algorithms.

H. P Wang[5] designed an automated stowage expert system with knowledge inference techniques, and implementation principles are provided. Kang et al and Kim et al[6-7] solved stowage planning problem with greedy algorithm and tree search algorithm. Ambrosino and Sciomachen[8] proposed a decision support system to solve Master Bay Plan Problem(MBPP), feasible solution was found by constraint satisfaction method, but objective function and optimal solution was not provided. Winter et al[12] introduced a stowage planning problem combining loading plan, and a decision support system was proposed to optimize the balance of quae cranes. Cho et al[13] and Botter[14] proposed a LP model for container ship stowage planning problem, some assumptions was made to simplify the problem. The planning result shows a weak compatibility to actual operation process. 
Avriel et al[15-16] proposed a 0-1 integer programming model, a heuristic method was designed to solve this problem. Numerical experiment with actual data shows an acceptable solution quality and computing efficiency. But this heuristic method is not without flaws, it can only solve simplified stowage planning problem and lacks flexibility. Ambrosino et al[17] proposed a model minimizing total loading time, with some operational constraints such as container type and load limit. The solution allocates containers with same port of discharge into same bay to avoid unnecessary restow. Sciomachen and Tanfani[18] solved stowage planning problem with 3D-BPP, optimizing total loading time and quae crane utilization. Pacino et al[19] and Delgado et al[20] solved stowage planning problem with two steps. The first step separates containers into different categories based on Master Bay Plan, and the second step decides the specific stowage location for each container.

These researches focuses mainly on optimizing ship stability, equipment utilization and total loading time. Other impact factors such as reshuffling operation and yard crane shifting are ignored. In practical application, stowage planning is a two-step process. In the first step, ship stability constraint is claimed and solved by ship line, and the result of the first step so called preplan is the input and constraint of the second step. This research focuses on the second step, which optimizes costs of container terminal, on condition that the constraint of the first step's results are satisfied. Formal researches about the second step often scales down the whole problem into ship bay stowage plan, for the independence of different bays of ship in stowage planning process. In practice, containers of the same bay and with the same key feature(container type, container size, port of discharge) are called a container group. In bay-wise stowage planning, decisions of different container groups are relative independent, thus a bay-wise stowage planning can be separated into several sub-bay-wise or so called group-bay planning problem. Therefore, a group-bay stowage planning model optimizing key cost factors of container terminal considering preplan constraints and operation constraints is proposed to solve stowage plan problem. A hybrid $A^{*}$-genetic algorithm is introduced to solve this problem. The stowage location is solved by the genetic phase and the loading sequence is updated by $A^{\star}$. Numerical experiments verifies the validity of the algorithm. This model and algorithm can be implemented to make stowage plans automatically.

\section{GROUP-BAY STOWAGE PLANNING PROBLEM ANALYSIS}

\section{INTRODUCTION OF STOWAGE PLANNING PROBLEM}

Planning a ship's stowage is a two-step process[1-2]. The first step is executed by the shipping line, so called pre-plan. This pre-planning phase optimizes general loading positions for each container group (containers with same POD, container type, size, status and height) from the shipping line's point of view to minimize the ship's utilization. Constraints contains mainly the stability of the ship. The second step is executed by container terminals, so called terminal-plan. This planning

phase optimizes specific slots and loading sequences for dedicated containers from the container terminal's point of view. Constraints are the result of the first step, and some operation constraints. Group-bay stowage planning is a part of the second step.

\section{DEFINITION OF GROUP-BAY STOWAGE PLANNING}

A container group is a set of containers with same POD, container type, size, status and height.

As is shown in Fig.1, the pre-plan loading positions of container group A are distributed in to ship bays, thus this container group A can be subdivided into two sets $02 \mathrm{H}-\mathrm{A}$ and $06 \mathrm{H}-\mathrm{A}$. These subsets called group-bays are the smallest decision unit in the terminal planning problem. In groupbay stowage planning problem, constraints are stability of the ship reflected in pre-plan(mainly the weight distribution constraints) and operation constraints.

$02 \mathrm{H}$

\begin{tabular}{|c|c|c|c|c|c|}
\hline & & & & & \\
\hline & & & & & \\
\hline A & A & A & A & A & A \\
\hline A & A & A & A & A & A \\
\hline A & A & A & A & A & A \\
\cline { 2 - 5 } & A & A & A & A & \multicolumn{2}{|c}{} \\
\cline { 2 - 5 } & &
\end{tabular}

Fig.1 Group-bays of a pre-plan

\section{OPTIMIZATION OBJECTIVES IN GROUP-BAY STOWAGE PLANNING}

(1) Reshuffles: In the loading process, if the previous loading container locates right under the subsequent loading container, the subsequent loading container must be removed from its row first to operate the previous loading container, this is called reshuffle.

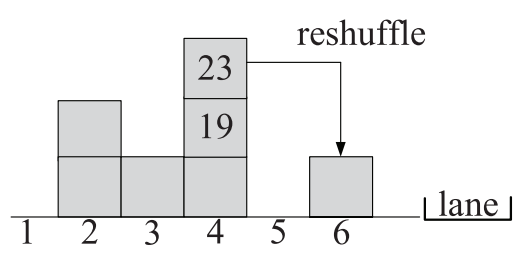

Fig. 2 Reshuffles in yard

In Fig.2, two containers are located in the same row of a yard bay. The container with sequence 19 is located right under the container with sequence 23 . When the yard crane carries containers in sequence, the 19th container should be transferred before the 23th container, and at that time, the 23th container must be reshuffled to another available location for the yard crane to get 19 th container. Reshuffles cost time 
and resources.

(2) Yard crane shifts: refers to yard crane movements between yard bays. A yard crane move from bay A to bay B is a shift.

Containers of same ship bay often locate in different yard bays, the loading sequence must be optimized to reduce yard crane shifts. Fig. 3 shows that an inferior loading sequence can cause frequent shifts, which costs time and resources.

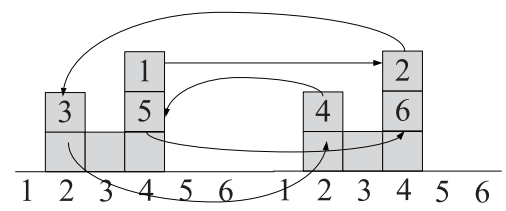

Bay:12 Bay:28

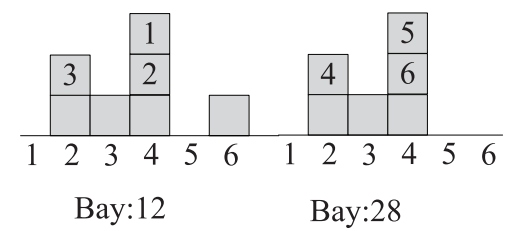

Fig.3 Yard crane shifts between different loading sequence

(3) Ship stability: In pre-plan optimization, container weight is a key decision factor. In terminal stowage planning, the final weight distribution must be as close as possible as the optimal weight distribution to ensure a feasible ship stability and seaworthiness.

\section{GROUP-BAY STOWAGE PLANNING MODEL}

Group-Bay stowage planning problem is a core and difficult planning problem of all planning in container terminals. This problem is a multi-objective combinatorial optimization problem with complex constraints. The major decision context is the specific loading location of each container and loading sequences of these containers. The constraints mainly embodies operation restrictions and ship stability control.

\section{MODEL ASSUMPTIONS}

1. The export containers to be stowed are no less than ship slots to be allocated;

2. The specific location of each container to be stowed is known;

3. The type of each container to be stowed is the same as the ship slots to be allocated;

4. Equipment failure is ignored;

5. Enough horizontal transportation trucks available;

6. The quae crane handles only one container per move.

\section{SYMBOL DEFINITION}

\section{(1) Dimensions}

$I$ : set of all containers to be stowed, $i, i^{\prime} \in I$;
$J$ : set of all ship slots to be allocated, $j, j^{\prime} \in J$;

$S$ : set of all loading sequences, $s \in S$;

$R$ : set of rows of ship slots to be allocated, $r, r^{\prime} \in R$;

$T$ : set of tiers of ship slots to be allocated, $t \in T$;

(2) Parameters

$A_{j r}$ : Whether ship slot $j$ is in ship row $r$, equals 1 if ship slot $j$ is in ship row $r$, otherwise 0 ;

$G P_{i}$ : Whether container $i$ is a GP container, equals 1 if container $i$ is a GP container, otherwise 0 ;

$H C_{i}$ : Whether container $i$ is a HC container, equals 1 if container $i$ is a $\mathrm{HC}$ container, otherwise 0 ;

$G P_{r}$ : Number of GP containers allowed in ship row $r$;

$H C_{r}$ : Number of HC containers allowed in ship row $r$;

$W_{j}$ : Minimum container weight of tier of ship slot $j$ according to pre-allocation according to pre-plan;

$M_{j}$ : Maximum container weight of tier of ship slot $j$ according to pre-allocation according to pre-plan;

$w_{i}$ : Weight of container $i$

$\delta$ : Maximum weight difference of heavier containers above lighter containers allowed in each ship row;

$Q_{s}$ : Matrix of serial numbers of loading sequences;

$Y P_{i i^{\prime}}$ : 0-1 Matrix, relative location of container $i$ and $i$, equals 1 if $i$ locates right above $i^{\prime}$ in yard, otherwise 0 ;

$V P_{i j^{\prime}}$ 0-1 Matrix, relative location of ship slots $j$ and $j^{\prime}$, equals 1 if $j$ locates right above $j$ ' in yard, otherwise 0 ;

$Y B_{i}$ : Yard bay of container $i$;

$F_{j}$ : Pre-allocation weight of ship slot $j$ according to pre-plan;

(3) Decision variables

$X_{s i j}$ : 0-1 variable, whether container $i$ is allocated to ship slot $j$ with loading sequence $s, 1$ for true, 0 for false;

$\beta_{i i^{\prime}}$ : 0-1 variable, relative loading sequence relation of container $i$ and $i^{\prime}$, equals 0 if container $i$ is to be loaded earlier than $i^{\prime}$, otherwise 1 ;

$\phi_{s}: 0-1$ variable, whether containers with consecutive loading sequence locates at the same yard bay, 1 for true, 0 for false;

\section{OBJECTIVE FUNCTION}

\section{(1) Minimize reshuffles}

Expression 3-1 is the relative loading sequence of container $i$ and $i^{\prime}$, if $i$ is to be loaded earlier than $i^{\prime}$ then $\beta_{i i^{\prime}}$ equals 0 , otherwise 1 .

$$
\beta_{i i^{\prime}}= \begin{cases}0, & \sum_{s \in S} \sum_{j \in J} X_{s i j} Q_{s}-\sum_{s \in S} \sum_{j \in J} X_{s i{ }^{\prime} j} Q_{s}<0 \\ 1, & \sum_{s \in S} \sum_{j \in J} X_{s i j} Q_{s}-\sum_{s \in S} \sum_{j \in J} X_{s i j} Q_{s}>0\end{cases}
$$

Thus the number of reshuffles is shown in expression 3-2:

$$
\min Z_{1}=\sum_{i, i^{\prime} \in I} Y P_{i i^{\prime}} \beta_{i i^{\prime}}
$$

(2) Minimize yard crane shifts

Expression 3-3 is whether the yard crane needs to shift from container $s-1$ to $s, 1$ for true, 0 for false. 
$\phi_{s}= \begin{cases}0, & \sum_{i \in I} \sum_{j \in J} X_{s i j} \cdot Y B_{i}-\sum_{i \in I} \sum_{j \in J} X_{(s-1) i j} \cdot Y B_{i}=0 \\ 1, & \sum_{i \in I} \sum_{j \in J} X_{s i j} \cdot Y B_{i}-\sum_{i \in I} \sum_{j \in J} X_{(s-1) i j} \cdot Y B_{i} \neq 0\end{cases}$

Thus, minimizing total yard crane shifts can be expressed as:

$$
\min Z_{2}=\sum_{s \in S} \phi_{s}
$$

(3) Minimizing total weight gap between planned containers and optimal weight distribution

$$
\min Z_{3}=\sum_{j \in J}\left|\sum_{s \in S} \sum_{i \in I} X_{s i j} \cdot w_{i}-F_{j}\right|
$$

\section{(4) Overall objective}

These three sub objects are all cost objects, the overall objective should cover the interaction of sub objects. According to practical handing cost measures, these sub objects can be treated as costs without normalizing. After consulted stowage planning stuff of Ningbo Daxie Container Terminal, a weighted overall function is given as follow:

$$
Z_{\text {total }}=0.5 Z_{1}+0.2 Z_{2}+0.3 Z_{3}
$$

\section{CONSTRAINTS}

\section{(1) Uniqueness constraint}

A container can only be allocated to a ship slot with only one loading sequence:

$$
\begin{gathered}
\sum_{s \in S} \sum_{j \in J} X_{s i j}=1 \\
\sum_{s \in S} \sum_{i \in I} X_{s i j}=1 \\
\sum_{i \in I} \sum_{j \in J} X_{s i j}=1
\end{gathered}
$$

\section{(2) GP and HC constraint}

In ship handling process, GP and HC containers are mixed in a ship row, which means in stowage planning, GP and HC containers are only restricted in numbers in quantity in a ship row, not in specific ship slots. HC quantity restriction of a ship row can be expressed as:

$$
\sum_{s \in S} \sum_{i \in I} \sum_{j \in J} X_{s i j} A_{j r} H C_{i}=H C_{r}
$$

$\mathrm{HC}$ quantity restriction of a ship row can be expressed as:

$$
\sum_{s \in S} \sum_{i \in I} \sum_{j \in J} X_{s i j} A_{j r} G P_{i}=G P_{r}
$$

\section{(3) Slot Weight limit}

According to optimal weight distribution based on pre-plan, slot weight limit is applied with a certain extent of relaxation. Container allocated to a slot must weights in this rang:

$$
W_{j} \leq \sum_{s \in S} \sum_{i \in I} X_{s i j} w_{i} \leq M_{j}
$$

(4) Maximum weight difference of heavier containers above lighter containers limit

To ensure the stability of the ship, the containers located above others should be lighter or a little bit heavier than the beneath container. This limitation can be expressed as:

$$
\left(\sum_{s \in S} \sum_{i \in I} X_{s i j} w_{i}-\sum_{s \in S} \sum_{i \in I} X_{s i j^{\prime}} w_{i}\right) V P_{j j^{\prime}} \leq \delta
$$

(5) Handling sequence constraint

The lower ship slots should be loaded before the upper ship slots, which means a container cannot be loaded to a ship slot above a not yet loaded ship slot.

$$
\sum_{s \in S} \sum_{i \in I} X_{s i j} Q_{s} \leq \sum_{j^{\prime} \in J}\left[\sum_{s \in S} \sum_{i \in I}\left(X_{s i j} Q_{s}\right) V P_{j j^{\prime}}\right]
$$

\section{ALGORITHM FOR GROUP-BAY STOWAGE PLANNING}

\section{INTRODUCTION OF HYBRID ALGORITHM}

A hybrid algorithm combining GA and $\mathrm{A}^{*}$ is proposed to solve this planning problem. In this algorithm, the genetic part is dedicated to solve the allocation of containers to ship slots, and the $\mathrm{A}^{\star}$ part solves the most feasible loading sequence of these containers to this allocation. The work flow of this algorithm is shown in Fig.4.

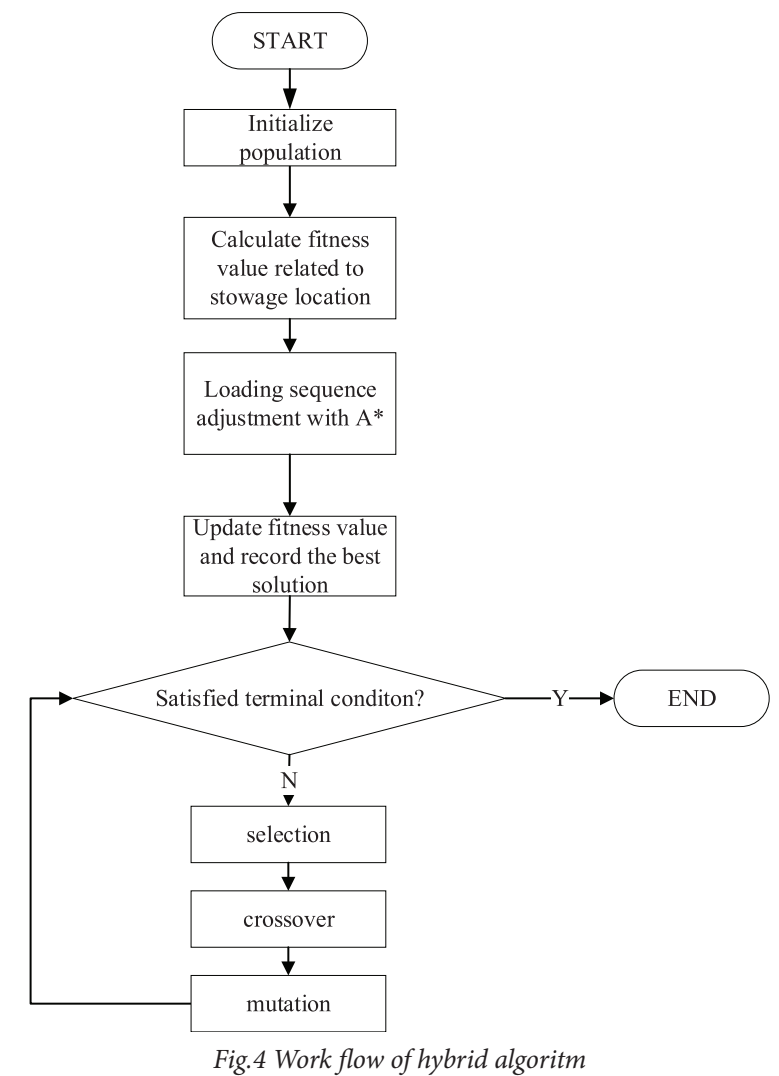




\section{CHROMOSOME DESIGN}

Fig. 5 shows a Chromosome of the genetic part, a gene is a ship slot for a container.

\begin{tabular}{|l|l|l|l|l|l|l|l|}
$\mathrm{A}$ & \multicolumn{1}{c}{$\mathrm{B}$} & $\mathrm{C}$ & $\mathrm{D}$ & $\mathrm{E}$ & $\mathrm{F}$ & $\mathrm{G}$ & $\mathrm{H}$ \\
\hline 2 & 1 & 8 & 4 & 7 & 5 & 3 & 6 \\
\hline
\end{tabular}

Fig.5 Example of a chromosome

\section{SELECTION}

GA part selects individuals to breed new generation with Roulette, the probability of individuals to be chosen to the new generation is calculated by fitness-based function:

$$
p_{i}=f_{i} / \sum_{i=1}^{n} f_{i}
$$

$p_{i}$ is the probability that $i$ is chosen to the new generation, $f_{i}$ is the fitness of $i, n$ is the population quantity.

\section{GENETIC OPERATORS}

Fig.6(a) shows that an order crossover(OX) operator is applied to make sure the generated chromosome has no ship slot repeat conflicts, but the GP and HC quantity may be infeasible. Therefore the GP and HC quantity constraint is converted into a penalty function to eliminate infeasible chromosomes during selection process.

Fig.6(b) is the mutation operator. This operator selects two different genes of one chromosome and switch them.

These genetic operators adjusts only the chromosome of slot allocation, not the loading sequence. The loading sequence is optimized trough the $\mathrm{A}^{*}$ part.

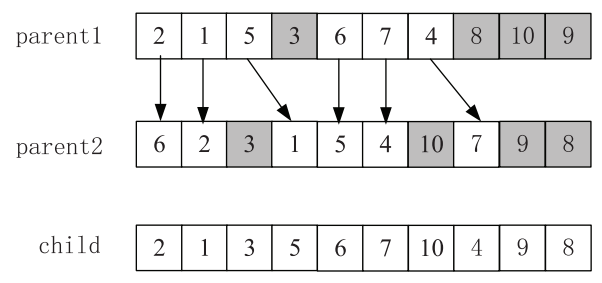

(a) Order crossover

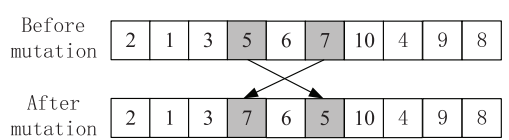

(b) Mutation

Fig.6 Genetic operators

\section{$A^{\star}$ FOR LOADING SEQUENCE ADJUSTMENT.}

This $\mathrm{A}^{\star}$ part adjusts loading sequence to minimize yard crane shifts after the genetic operators allocates the slots.

$$
Z_{2}(i)=g_{2}(i)+h_{2}(i)
$$

Expression 4-16 shows that the cost function consists of two parts: $g_{2}(i)$ is the actual cost from start node to loading container $i$, which is total yard crane shifts until loading container $i ; h_{2}(i)$ is the estimated minimum yard crane shifts after container $i$ is loaded till the end of the loading, which is the total number of yard bays who have containers to be loaded after container $i$.

Fig.7 shows the expansion of child node of $A^{\star}$, a constraint branch pruning is introduced to eliminate infeasible solution. In the searching process, these gray slots are slots that can be loaded according to handling sequence constraint, which means only these gray slots are feasible child nodes. This $\mathrm{A}^{*}$ part expands feasible nodes from start node and calculates the cost function, and selects the node with the least cost function as the current node to expand, and the final loading sequence is get through iterations. After loading sequence searching for each chromosome, the fitness function is calculated according to the slot allocation and the loading sequence to get the selection probability of each chromosome.

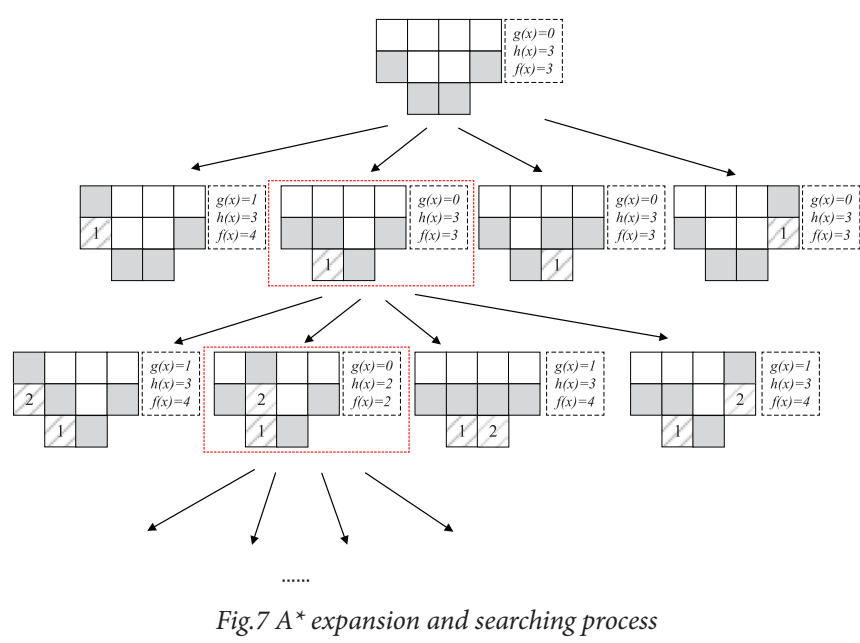

NUMERICAL EXPERIMENT

In this part, a numerical example from Ningbo Daxie Container Terminal is chosen to validate the feasibility of the proposed model and algorithm.

\section{NUMERICAL EXAMPLE}

This numerical is $30 \mathrm{H}$ bay of a container ship. The 42 containers to be stowed distributes in 6 yard bays. Fig. 8 shows the pre-plan of this group-bay, blue slots are to be allocated to. Fig. 9 shows the containers and their yard locations and weight, yellow containers are to be allocated. The experiment platform is a computer with a dual core $2.50 \mathrm{GHz}$ Intel Core 
i7 CPU, 4.00GB RAM and a Win7 64bit OS.

$\begin{array}{llllllllllllllllll}18 & 16 & 14 & 12 & 10 & 08 & 06 & 04 & 02 & 01 & 03 & 05 & 07 & 09 & 11 & 13 & 15 & 17\end{array}$

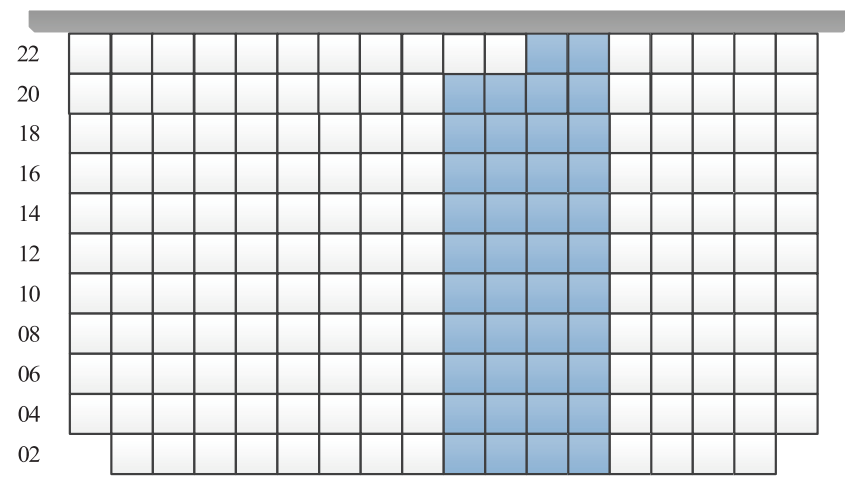

Fig.8 Pre-plan of bay $30 \mathrm{H}$

Group-Bay: NDLTM40GP-30H

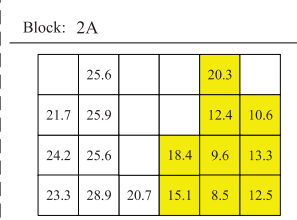

Bay: 06
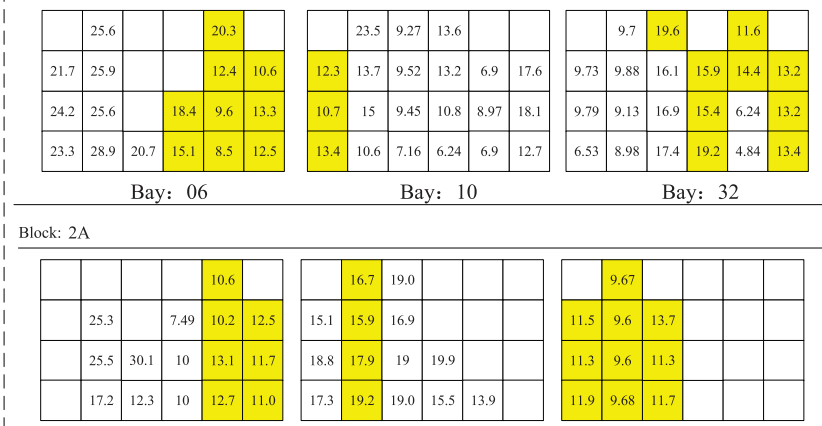

Bay: 10

Bay: 32
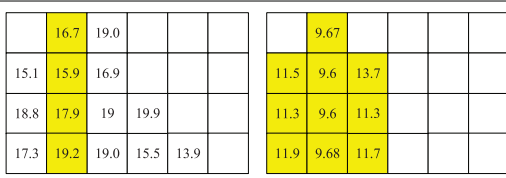

Bay: 62

Fig.9 Yard distribution of containers

\section{RESULTS AND ANALYSIS}

(1) Preset of Parameters

The parameter set is in Table.1:

Tab. 1 Parameter set of GA

\begin{tabular}{cccc}
\hline Population & Iterations & $\begin{array}{c}\text { Crossover } \\
\text { Prob. }\end{array}$ & Mutation Prob. \\
\hline 50 & 1000 & 0.85 & 0.05 \\
\hline
\end{tabular}

(2) Validation of proposed algorithm

The stowage plan of proposed algorithm is in Fig.10. Yard crane shifts in yard block $2 \mathrm{~A}$ according to loading sequence is $56 \rightarrow 10 \rightarrow 48 \rightarrow 06 \rightarrow 62 \rightarrow 32 \rightarrow 62$. Total reshuffle is 0 , total shifts are 6 , with only one shift back from yard bay 32 to yard bay 62. And all allocated containers satisfies the weight constraint and maximum weight difference of heavier containers above lighter containers constraint.

(3) Convergence of proposed algorithm

The convergence curve is Fig.11:

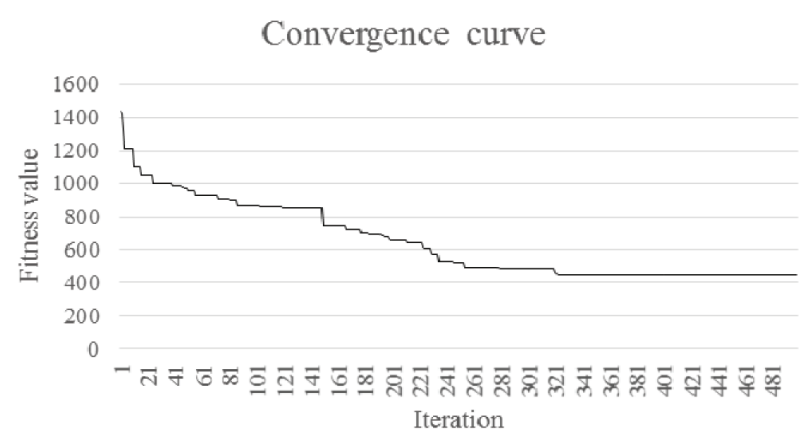

Fig.11 Convergence curve of proposed algorithm

$\begin{array}{llllllllllllllllll}18 & 16 & 14 & 12 & 10 & 08 & 06 & 04 & 02 & 01 & 03 & 05 & 07 & 09 & 11 & 13 & 15 & 17\end{array}$

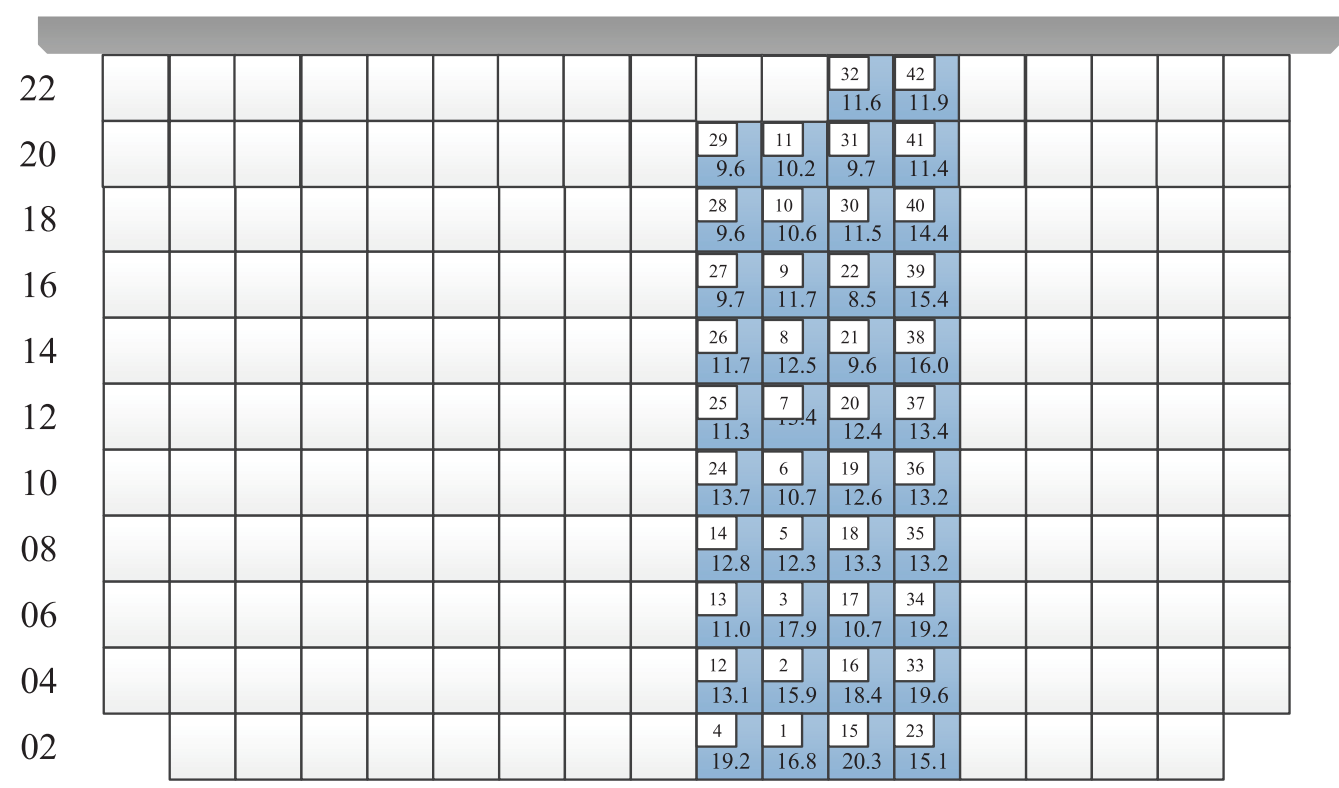

Fig.10 Stowage planning result by proposed algorithm 
As is shown in Fig.11, proposed algorithm converged to a near-optimal solution, the curve went flat afterwards, which shows an acceptable convergence.

To go a step further, the algorithm is run 30 times. Table. 2 is the result. Iterations to get near-optimal solution is the convergence speed.

\section{Tab.2 30 run time result of proposed algorithm}

\begin{tabular}{cc|cc|cc}
\hline NO. & $\begin{array}{c}\text { Iterations to get } \\
\text { near-optimal } \\
\text { solution }\end{array}$ & NO. & $\begin{array}{c}\text { Iterations to get } \\
\text { near-optimal } \\
\text { solution }\end{array}$ & NO. & $\begin{array}{r}\text { Iterations } \\
\text { to get near- } \\
\text { optimal } \\
\text { solution }\end{array}$ \\
\hline 1 & 322 & 11 & 504 & 21 & 485 \\
\hline 2 & 356 & 12 & 492 & 22 & 317 \\
\hline 3 & 467 & 13 & 412 & 23 & 587 \\
\hline 4 & 451 & 14 & 563 & 24 & 425 \\
\hline 5 & 332 & 15 & 395 & 25 & 536 \\
\hline 6 & 353 & 16 & 536 & 26 & 328 \\
\hline 7 & 289 & 17 & 529 & 27 & 489 \\
\hline 8 & 567 & 18 & 462 & 28 & 439 \\
\hline 9 & 351 & 19 & 551 & 29 & 595 \\
\hline 10 & 326 & 20 & 595 & 30 & 400 \\
\hline
\end{tabular}

For 30 independent experiments, the max iteration to get near-optimal solution is 595 , the min iteration is 289 , and the average is 448 . This result shows a relatively high convergence efficiency, and an acceptable stability. For all numerical examples tested, the proposed algorithm converges in 600 iterations, the parameter of 1000 total iterations is feasible, and the convergence of proposed algorithm is validated.

(4) Effectiveness of proposed algorithm

To verify effectiveness of proposed algorithm, a standard GA is used as control. The two algorithms use both 1000 iterations. Average iterations to get near-optimal solution, average fitness value, standard deviation of fitness and average solution time are listed in Table.3.

Tab.3 Comparison of proposed algorithm and GA

\begin{tabular}{ccccc}
\hline & $\begin{array}{c}\text { Average } \\
\text { iterations to get } \\
\text { near-optimal } \\
\text { solution }\end{array}$ & $\begin{array}{c}\text { Average } \\
\text { fitness } \\
\text { value }\end{array}$ & $\begin{array}{c}\text { Standard } \\
\text { deviation of } \\
\text { fitness }\end{array}$ & $\begin{array}{c}\text { Average } \\
\text { solution } \\
\text { time(s) }\end{array}$ \\
\hline $\begin{array}{c}\text { Proposed } \\
\text { hybrid } \\
\text { algorithm }\end{array}$ & 448 & 322.5 & 6.45 & 37.51 \\
\hline $\begin{array}{c}\text { Standard } \\
\text { GA }\end{array}$ & 729 & 584.2 & 22.39 & 59.28 \\
\hline
\end{tabular}

Table. 3 shows that proposed algorithm has advantage over standard GA in all four measures, although both algorithms can get near-optimal solution in given iterations, and this shows the performance of the proposed algorithm. Standard GA gets near-optimal solution in average 729 iterations against hybrid algorithm's 448 . This verifies the convergence speed superiority of proposed hybrid algorithm. The average value shows that proposed algorithm gets a much more global optimized result. The standard deviation of fitness advantage of proposed algorithm shows the stability advantage of hybrid algorithm over GA. And the hybrid algorithm has a $21.37 \mathrm{~s}$ advantage over GA in average solution time, which counts as has a better solution efficiency.

With better result and less time consumption, the propose algorithm can be considered to be a feasible algorithm for solving Group-bay stowage planning problem.

\section{CONCLUSIONS}

This study developed stowage planning problem in three major aspects:

(1) Defines group-bay stowage planning problem which is the smallest planning sub problem in the terminal planning problem.

(2) A MIP model of group-bay stowage planning problem optimizing yard reshuffles, yard crane shifts and weight distribution is introduced with operational principles.

(3) A GA-A* hybrid algorithm is proposed to solve both the stowage location allocation and loading sequencing of the group-bay stowage planning problem.

(4) Numerical experiment shows the convergence and effectiveness of the proposed algorithm in solving group-bay stowage planning problem.

In the synthesis, group-bay stowage planning problem can be solved through proposed algorithm, which provides a feasible solution and an aspect of view to the terminal stowage planning problem.

\section{REFERENCES}

1. Steenken, D., Voß, S., \& Stahlbock, R. Container terminal operation and operations research A classification and literature review. OR Spectrum, 26(1), 2004, 3-49.

2. Alvarez, J. Ambrosino, D., Anghinolfi, D., Paolucci, M A heuristic for vessel planning in a reach stacker terminal[J]. Journal of Maritime Research, III(1), 2006, 3-16.

3. Mi Chao, Zhang Zhiwei, Huang Youfang, Shen Yang, A FAST AUTOMATED VISION SYSTEM FOR CONTAINER CORNER CASTING RECOGNITION,Journal of Marine Science and Technology-Taiwan, 2016, 24(1): 54-60. DOI: 10.6119/JMST-016-0125-8

4. Mi Chao, Shen Yang, Mi Weijian, Huang Youfang, Ship Identification Algorithm Based on 3D Point Cloud for Automated Ship Loaders, Journal of Coastal Research, 2015, 2015(SI.73):28-34. DOI: 10.2112/SI73-006.1 
5. Wang Hongpeng. Knowledge-based Container Ship Automatic Stowage Expert System [J]. Journal of Shanghai Maritime University,2002,23(1):46-49.

6. Kang, J.-G., \& Kim, Y.-D. Stowage planning in maritime container transportation[J]. Journal of the Operational Research Society, 53(4), 2002, 415-426.

7. Kim, K. H., Kang, J. S., \& Ryu, K. R. A beam search algorithm for the load sequencing of outbound containers in port container terminals[J]. OR Spectrum, 26(1), 2004, 93-116.

8. Ambrosino D., Sciomachen A.. A constraints satisfaction approach for master bay plans[J]. In: Sciutto, G., Brebbia, C. (Eds.), Maritime Engineering and Ports. WIT Press, Boston, 1998, pp: 155-164.

9. Mi Chao, Zhang Zhiwei, He Xin, Huang Youfang, Mi Weijian, Two-stage classification approach for human detection in camera video in bulk ports, Polish Maritime Research, 2015, 22(SI.1):163-170. DOI: 10.1515/ pomr-2015-0049

10. Mi Chao, He Xin, Liu Haiwei, Huang Youfang, Mi Weijian, Research on a Fast Human-Detection Algorithm for Unmanned Surveillance Area in Bulk Ports, Mathematical Problems in Engineering, 2014, Article No.386764.DOI: $10.1155 / 2014 / 386764$

11. Mi Chao, Liu Haiwei, Huang Youfang, Mi Weijian, Shen Yang, Fatigue alarm systems for port machine operators, ASIA LIFE SCIENCES, 2016,25(1):31-41.

12. Winter T.. Online and Real-Time Dispatching Problems[D], PhD thesis, Technical University of Braunschweig, Germany, 1999.

13. Cho D.W.. Development of a methodology for containership load planning[D], PhD thesis, Oregon State University, 1984.

14. Botter R.C., Brinati M.A.. Stowage container planning: a model for getting an poptimal solution[J],IFIP Trans. B (App. in Tech.), 1992, B-5: 217-229.

15. Avriel M., Penn M.. Exact and approximate solutions of the container ship stowage problem[J]. Computers \& Industrial Engineering, 1993, 25: 271-274.

16. Avriel M., Penn M., Shpirer N., Witteboon S.. Stowage planning for container ships to reduce the number of shifts[J]. Annals of Operations Research, 1998, 76: 55-71.

17. Ambrosino D., Sciomachen A.,Tanfani E.. Stowing a containership: the master bay plan problem[J]. Transportation Research A, 2004, 38(2): 81-99.
18. Sciomachen A., Tanfani E.. A 3D-BPP approach for optimising stowage plans and terminal productivity[J]. European Journal of Operational Research, 2007, 183: $1433-1446$.

19. Pacino, D., Delgado, A., Jensen, R. M., \& Bebbington, T. Fast generation of near-optimal plans for eco-efficient stowage of large container vessels. In Proceedings of the second international conference on computational logistics, 2011, ICCL'11 (pp. 286-301), Berlin, Heidelberg: Springer-Verlag.

20. Delgado, A., Jensen, R. M., Janstrup, K., Rose, T. H., \& Andersen, K. H. A constraint programming model for fast optimal stowage of container vessel bays. European Journal of Operational Research, 2012, 220, 251-261.

\section{CONTACT WITH THE AUTHOR}

\author{
Mi Weijian \\ Logistics Engineering College \\ Shanghai Maritime University \\ 201306, Shanghai \\ CHINA
}

